\title{
Polyphenols in cancer prevention: New insights (Review)
}

\author{
GIUSI BRIGUGLIO $^{1}$, CHIARA COSTA ${ }^{2}$, MANUELA POLLICINO ${ }^{1}$, \\ FEDERICA GIAMBÒ $^{1}$, STEFANIA CATANIA ${ }^{1}$ and CONCETTINA FENGA ${ }^{1}$ \\ ${ }^{1}$ Department of Biomedical and Dental Sciences and Morpho-functional Imaging, Occupational Medicine Section, \\ and ${ }^{2}$ Department of Clinical and Experimental Medicine, University of Messina, I-98125 Messina, Italy
}

Received July 30, 2020; Accepted September 21, 2020

DOI:10.3892/ijfn.2020.9

\begin{abstract}
A huge volume of literature data suggests that a diet rich in fruits and vegetables, mostly due to the contribution of natural polyphenols, could reduce the incidence of specific cancers. Resveratrol, epigallocatechin gallate and curcumin are among the most extensively studied polyphenols: The majority of the effects attributed to these compounds are linked to their antioxidant and anti-inflammatory properties. The multiple mechanisms involved include the modulation of molecular events and signaling pathways associated with cell survival, proliferation, differentiation, migration, angiogenesis, hormonal activities, detoxification enzymes and immune responses. Notwithstanding their promising role in cancer prevention and treatment, polyphenols often have a poor bioavailability when administered as pure active principles, representing an important limit to their use. However, the bioavailability and thus the efficacy of these compounds can be improved by their administration in combination with other phytochemicals, with anticancer drugs or in polyphenol-loaded nanotechnology-based delivery systems. The possibility of combining conventional drugs with polyphenols offers very valuable advantages, such as the building of more efficient anticancer therapies with less side-effects on the health of patients. The present review focuses on current knowledge regarding the interactions between natural polyphenols and cancer development in order to gain a clearer comprehension of the potential mechanisms through which individual foods and food components may be exploited to reduce cancer risk.
\end{abstract}

Correspondence to: Professor Concettina Fenga, Department of Biomedical and Dental Sciences and Morpho-functional Imaging, Occupational Medicine Section, University of Messina, Policlinico Universitario 'G. Martino' - pad. H, Via Consolare Valeria 1, I-98125 Messina, Italy

E-mail: cfenga@unime.it

Key words: polyphenols, cancer, oxidative stress, resveratrol, curcumin, epigallocatechin gallate, diet

\section{Contents}

\author{
1. Introduction \\ 2. Literature search \\ 3. Classification of polyphenols \\ 4. Prostate cancer \\ 5. Colon cancer \\ 6. Breast cancer \\ 7. Lung cancer \\ 8. Bladder cancer \\ 9. Skin cancer \\ 10. Pancreatic cancer \\ 11. Leukemia \\ 12. Conclusion
}

\section{Introduction}

Cancer is considered to be one of the most serious issues affecting humanity worldwide and is the second leading cause of mortality after cardiovascular diseases (1). The World Health Organization predicts that by 2050 there will be approximately 27 million cases with an annual mortality rate of 17.5 million individuals (2).

The onset of cancer can be influenced by a number of factors, such as genetic, metabolic, occupational and environmental including benzene, heavy metals, chemicals and mineral fibers (3-5).

These factors can act directly or indirectly, stimulating the expression of the malignant phenotype that regulates its progression. In particular, approximately 30-35\% of cancer cases are associated with dietary factors: There is clear evidence suggesting the role of diet in controlling and/or promoting cancer. Combining this evidence, the Mediterranean diet represents one of the most popular dietary models currently under consideration in the field of cancer prevention and treatment outcomes (6). A lower incidence of cancer has been observed in countries where eating habits involve a low intake of meat, moderate intake of dairy and alcohol, and the frequent consumption of fruit and vegetables rich in bioactive nutrients (7). In fact, thousands of phytochemicals with antioxidant, anti-inflammatory, anticarcinogenic, antiviral and antiallergic properties are present in fruits and vegetables; they are classified as carotenoids, vitamins, alkaloids, nitrogen-containing, organosulfuric and phenolic compounds (1). In particular, 
in vivo and in vitro analyses, as well as human studies, have suggested the protective effects of polyphenols against chronic diseases and have demonstrated an inverse association between the consumption of polyphenols and the risk of tumor development.

A study published in the Cochrane Database of Systematic Reviews assessed possible associations between green tea consumption and the risk of cancer incidence and mortality, as well as safety data and quality of life as secondary outcomes. The authors reviewed 11 experimental studies, including a total of 1,795 participants consuming either green tea extract or a placebo and 131 non-experimental studies $(>1,100,000$ participants) (8). A beneficial effect due to green tea consumption emerged only for the high-intake subjects at specific cancer sites from randomized control trials and case-control studies; however methodological limitations, such as the low number and size of the studies as well as inconsistency with the results of cohort studies, limit the interpretability of the relative risk estimates.

Phenolic compounds act on carcinogenesis throughout the induction of cell defense systems, including detoxifying and antioxidant enzyme systems, as well as the inhibition of the anti-inflammatory and anti-cellular growth signaling pathways that culminate in cell cycle arrest and/or cellular death. These contributions strongly suggest the anticancer effects of polyphenols, due to their ability to alter the epigenome of cancer cells (2,9-14). Specifically, natural polyphenolic compounds present in the diet can exert their anticancer effects through a variety of mechanisms, including the modulation of cell cycle signaling, the removal of anticancer agents, the activity of antioxidant enzymes, apoptosis and arrest of the cell cycle.

Recent in vitro studies have suggested that these compounds modulate Nrf2 and NF- $\mathrm{KB}$ activation in cells and can significantly influence MAPK and PI3K function in cells, demonstrating their role in the proliferation of cancer cells $(15,16)$. Moreover, natural polyphenols, e.g., apigenin, resveratrol (RES), genistein, luteolin and quercetin have been shown to induce the apoptosis of several malignant cells $(2,17)$.

Numerous protein growth factors can induce endothelial cell proliferation and angiogenesis by producing various inflammatory mediators and lipid second messengers, such as prostaglandins and platelet-activating factor (PAF). PAF is a potent mediator of inflammation that is implicated in several pathological conditions, including cancer. In particular, tumor cells, as well as activated endothelial cells expose the PAF receptor on their membrane surface. When PAF binds to its receptor, numerous processes are activated that determine the onset and development of tumor-induced angiogenesis and metastases. The Mediterranean diet provides a dietary profile characterized by the presence of antioxidant substances and PAF inhibitors. Consequently, it can have preventive and protective effects against the development, growth and metastatic manifestations of cancer, through the inhibition of PAF activity and/or its biosynthesis $(18,19)$ The main antiplatelet effect of flavonoids may be due both to the inhibition of thromboxane formation and to the antagonism of the thromboxane receptor (20). In addition, RES and tyrosol have been shown to exhibit biological activity as inhibitors of PAF-induced platelet aggregation (21).
Oxidative stress and DNA damage are the most common triggers activating the mitochondrial apoptotic pathway, which can result in mitochondrial membrane breakage and the release of cytochrome $c(22)$.

In addition, other dietary compounds, such as zinc and folate are involved in the DNA repair process, although they do not share an identical mechanism. Some of these have been shown to play a role in epigenetics, while others may interact with genes other than those directly involved in DNA repair and methylation. Some play a protective role against oxidative stress, while others inhibit cell proliferation by modifying the inflammatory process. For example, there is an association between folate deficiency and colorectal cancer, although the risk varies due to genetic interindividual variability (23).

There is increasing evidence to indicate that the consumption of cruciferous vegetables reduces the risk of lung and colorectal carcinoma among subjects with low manifestation of enzymes responsible for the biotransformation of sulforaphane, the actual bioactive compound. These examples are some of the numerous associations between nutrients/genes/cancers (10).

On this basis, the present review focuses on current knowledge regarding the interactions between natural polyphenols and cancer development in order to gain a clearer comprehension of the potential mechanisms through which individual foods and food components may be exploited to reduce cancer risk.

\section{Literature search}

PubMed was searched to identify full text studies conducted on humans and published in the English language over the past year, which associate the dietary intake of polyphenols with the prevention of some prevalent cancer sites. Tumor types included in literature search were selected on the basis of their worldwide incidence. Some studies, although methodologically adequate, were excluded as they were not considered relevant to the study purpose.

The majority of citations were found using the terms: 'Polyphenols' AND 'Cancer' OR 'Prostate Cancer' OR 'Breast Cancer' OR 'Bladder cancer' or 'skin cancer' or 'blood cancer' or 'colon cancer' or 'lung cancer' or 'pancreatic cancer'. There were no restrictions applied on the country of origin, ethnicity or sex. The relevance of the subject and the admissibility of all the publications retrieved was further assessed on the basis of titles and abstract. Further relevant studies were identified through the manual screening of the reference lists of selected articles and recently published reviews.

\section{Classification of polyphenols}

Polyphenols (Table I) are mainly classified into flavonoids and non-flavonoids (24). They are natural compounds that are all derived from phenylalanine and contain an aromatic ring with one or more hydroxyl groups. They include a large class of antioxidants such as flavonoids, phenolic acids and their derivatives, lignans and stilbenes.

The principal phenolic acids include hydroxybenzoic acids (e.g., gallic, p-hydroxybenzoic, vanillic and syringic acid) and hydroxy-cinnamic acids (e.g., ferulic, caffeic, p-coumaric, chlorogenic and synapic acid); however, due to their structural 
Table I. Most representative polyphenols: Chemical formula and vegetal sources.

\begin{tabular}{lll}
\hline Polyphenols & Chemical formula & Vegetal sources
\end{tabular}

Resveratrol<smiles>Oc1ccc(/C=C/c2cc(O)cc(O)c2)cc1</smiles>

Licopen

Curcumin

Epigallocatenin gallate
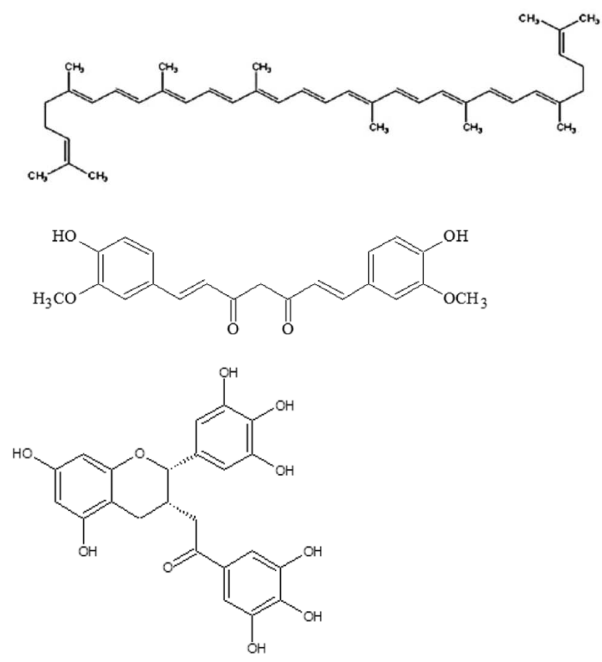

Oleuropein

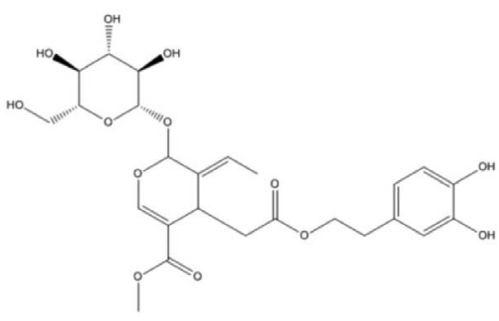

Red grapes, blueberries, berries

Tomatoes, strawberries, cherries, pomegranate, blood oranges, watermelon, papaya

Turmeric

White, green and black tea, apples, blackberries, raspberries, pecans, hazelnuts, peaches, avocados, pistachios and onions

Olive, olive oil similarity, many other polyphenols are considered analogues of phenolic acid, such as capsaicin, rosmarinic acid, gingerol and gossypol. Tea is an important source of gallic acid, whereas the richest sources of hydroxy-cinnamic acids are coffee, lettuce, carrots, berries, sweet potatoes, prunes, peaches, apples, tomatoes and grapes.

Flavonoids are the most abundant polyphenols in diet. They are classified into flavones, flavonols, flavanols, flavanones, isoflavones and anthocyanins. These compounds have the basic skeleton of phenylbenzopyrone consisting of 2 aromatic rings. In nature, flavonoids can occur both in free and conjugated form. Among the predominant flavonols, quercetin is mentioned. The main food sources containing them are onions, cherries, apples, broccoli, cabbage, tomatoes, berries, tea, red wine, cumin and buckwheat.

Flavanones are mainly found in citrus fruits (e.g., oranges, lemons and aurantium), grapes and the medicinal herbs of Rutaceae, Rosaceae and Leguminosae.

Flavanols such as catechin, epicatechin, epigallocatechin, epicatechin gallate and epigallocatechin gallate (EGCG), are also widespread in medicinal herbs and diet plants (e.g., tea, apples, berries, cocoa and catechu). The main sources of flavones (luteolin, apigenin and tangeritin) are leaves, rinds, barks and pollens. Isoflavones include daidzein, genistein, glycitein, found in soybeans and other legumes. Anthocyanidines are a typically colored group of flavonoids; they are present in flowers and red, blue or purple fruits. Within the subclass of stilbenes, RES has been found in several edible natural products such as grapes, peanuts, berries and rhubarb (25).

\section{Prostate cancer}

Prostate cancer (PC) represents the second most commonly diagnosed cancer among males worldwide (26). Countries following a Mediterranean-type dietary pattern (Italy, Greece, Spain, Malta and some regions of France) have been documented to have a lower incidence and mortality rate due to PC than northern European regions. In a population-based case-control study conducted in Southern Italy, a total of 118 patients with PC and 238 controls were examined; the controls had a significantly higher adherence to the Mediterranean diet, following correction for confounding factors as age, body mass index, cigarette smoke, alcohol intake and physical activity (27).

The lowest age-standardized incidences of PC worldwide are registered in South Central Asia compared to westernized countries; lifestyle choices, including diet are considered to be involved in the risk of developing PC, as known risk 
factors such as age, race and family history cannot explain this geographical variability in incidence. In fact, the risk of developing PC rapidly increases (as much as 20-fold) in Asian immigrants to the United States who reduce their intake of soy, tea, fish, fruits and vegetables, while introducing more red meat and fat (28).

Therefore, dietary components such as vegetables, tomato sauce, fish and vegetable fat are associated with a lower risk of PC progression. In particular, dietary factors that can influence the onset of PC are high intake of alcohol, dairy products, animal fat and meat; lycopene also has a tendency towards a favorable effect on PC incidence. On the other hand, the intake of long-chain omega-3 polyunsaturated fatty acids does not seem to be associated with lower cancer incidence (26). Vitamin D could also play an important role, as both low and high concentrations are associated with an increased risk (29).

In light of these considerations, scientific interest in the potential chemopreventive role in PC of various phytochemicals present in food is increasing. The mechanisms through which they exert their anticancer activity include the inhibition of proliferation, the induction of apoptosis and arrest of the cell cycle. Moreover, they can modulate signaling pathways and influence epigenetic alterations, such as DNA methylation and expression patterns of microRNAs (miRNAs). In particular, some polyphenols (lycopene, EGCG, CUR) act through the downregulation of different signal transduction pathways. Also sulphoraphane seems to exert antitumor effect through several mechanisms, such as anti-inflammatory, anti-angiogenetic and anti-metastatic activities (Fig. 1) (10).

Epidemiological studies have indicated that these plant bioactives exhibit multimodal effects on PC cells, and foods such as cruciferous and alliaceous vegetables, tomatoes, red wine, green tea, turmeric and pomegranate have all been linked to a reduced risk of developing PC. For example, red wine and grapes contain RES, which has various biological effects, such as anti-inflammatory, antioxidant and anticancer. RES inhibits the in vitro dehydrotestosterone-induced progression of PC, interfering with the AR and CXCR4 pathway. CXCR4 as a chemokine receptor has been found to be upregulated in cancer metastases and has been used as a prognostic marker in various types of cancer, including leukemia, breast cancer (BC) and PC: RES treatment reduced CXCR4, AR, p-PI3K and p-AKT expression (30).

Green tea polyphenols containing EGCG have also been shown to exhibit numerous noteworthy biological activities, including anticancer properties. In particular recent studies on PC have demonstrated that epigenetic mechanisms could represent the main actors in the regulation of matrix metalloproteinases (MMP) and their tissue inhibitor (TIMP) associated with the progression of PC. In vitro, GTP/EGCG mediates the epigenetic reactivation of TIMP-3, which acts by inhibiting PC progression. In addition, clinical trials performed on patients undergoing prostatectomy and consuming $800 \mathrm{mg}$ EGCG for up to 6 weeks, compared to matched controls, demonstrate an increase in plasma TIMP-3 levels. These findings confirm that epigenetic induction of TIMP-3 by green tea consumption restores the MMP/TIMP balance suppressing the progression of PC (31).

\section{Colon cancer}

Colorectal cancer (CRC) is the third most common type of cancer worldwide with a high incidence and mortality (32). $\mathrm{CRC}$ is recognized as a multifactorial disease that is dependent on environmental variables and individual intrinsic factors. In particular, eating habits have been linked to changes in the intestinal microbiota which could also contribute to the pathophysiology underlying CRC and its metabolic and psychological complications. The intestinal microbiota has a well-defined role in the body's homeostasis, in fact several highly prevalent gastrointestinal diseases have been associated with imbalances in microbiota composition (dysbiosis) and in particular the association with the onset of cancer has been suggested (33).

Among the bioactive compounds of the diet, polyphenols exert favorable effects on the intestinal microbiota, on free radicals and on inflammation (34). Polyphenols are biotransformed by the intestinal microbiota and finally metabolized into relatively simple aromatic carboxylic acids, commonly termed phenolic acids (35). These bioavailable metabolites can be even more bioactive than their precursors. However, only $5-10 \%$ of the total intake in polyphenols can be absorbed in the small intestine.

The modification of the composition of intestinal microbiota can significantly influence the bioavailability of polyphenols. In fact, human intestinal microbiota exhibits important hydrolytic activity, therefore when polyphenols reach the colon, their bioavailability can be significantly increased. In addition, polyphenols can decompose into smaller phenolic acids for easy absorption by the intestinal mucosa.

EGCG can be methylated through catechol- $O$-methyltransferase and can exert an inhibitory effect on DNA methyltransferase (DNMT). DNMT inhibition prevents hypermethylation of newly formed DNA strands, leading to a reversal process of silenced genes. EGCG may also suppress DNMT action by activating genes silenced by tumor cells by methylation (36).

An in vitro study performed on AK4-knockdown colon cancer SW480 and SW620 cells demonstrated that RES can inhibit the invasion and metastasis of colon cancer cells by reversing the expression of epithelial-mesenchymal transition (EMT) markers through the AKT/GSK-3 $\beta /$ Snail signaling pathway. In fact, AKT1 can be a key regulator of EMT in colon cancer cells and serve as a potential therapeutic target for this disease (37).

Djulis, a cereal crop rich in polyphenols and dietary fiber, can also prevent CRC. Indeed Lee et al carried out a study on rats showing that polyphenols can protect rats from oxidative stress and regulate proteins related to anti-apoptosis, proapoptosis and proliferation to prevent CRC progression. Therefore, djulis may prove to be a promising CRC chemopreventive product in the future (32).

Inflammation-induced carcinogenesis has been shown to be associated with oxidative stress, genomic instability, immune effectors, cytokine dysregulation and the modulation of the NF- $\kappa \mathrm{B}$ signaling pathway. Foods such as strawberries and black raspberries have been shown to play a synergistic role in multiple molecular events, including the suppression of cytokine release, the reduction of oxidative stress, the 


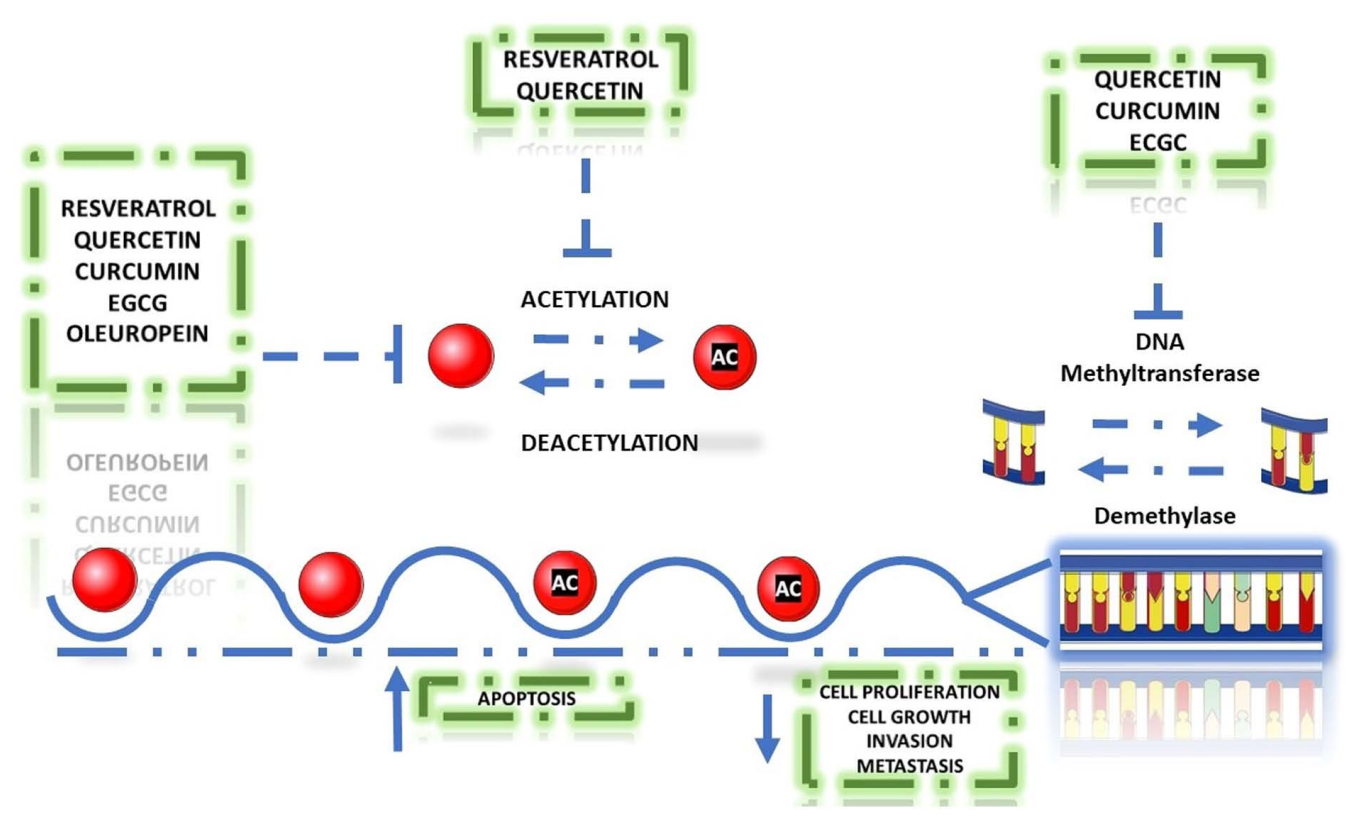

Figure 1. Polyphenols act on the epigenetic mechanism by inhibiting the methyltransferase activities of HDAC, HAT and DNA which are deregulated in cancer cells.

reduction of genomic instability and the inhibition of $\mathrm{NF}-\kappa \mathrm{B}$ and related pathways (38).

A study carried out by $\mathrm{Hu}$ et al demonstrated a synergistic action of ginkgetin and RES in preventing VEGF-mediated angiogenesis in several experimental models, suggesting that this combination may play a role in anti-tumor treatment. The reduced formation of sub-intestinal vessels was shown in zebrafish embryos and microvascular sprouting in rat aortic ring; the reduced phosphorylation of VEGFR2, Akt, eNOS and Erk, as well as the expression of matrix metalloproteinases (MMPs) was found in human umbilical vein endothelial cells (HUVECs). The combination of ginkgetin and RES was also effective on HT-29 colon cancer xenograft nude mice and relieved the 5-fluorouracil-induced inflammatory response by suppressing expression levels of COX-2 and inflammatory cytokines (39).

\section{Breast cancer}

$\mathrm{BC}$ is the second most frequent type of cancer worldwide; in particular, among women, it is considered the most commonly diagnosed type of cancer, constituting approximately $25 \%$ of all diagnosed tumors (7).

The risk of developing $\mathrm{BC}$ is affected by non-modifiable factors, including age and genotype, as well as modifiable factors, such as smoking, alcohol, nutrition and occupational exposure (40). It has been observed that obesity, a sedentary lifestyle and unhealthy diet are known to increase the mortality rate of BC survivors. As the same risk factors are common to cardiovascular diseases, the risk of cardiovascular diseases is also higher among $\mathrm{BC}$ survivors. Consequently, the implementation of a healthy diet rich in unrefined cereal, fresh fruit and vegetables could also indirectly improve the outcome of $\mathrm{BC}$ survivors by reducing body weight. The nutrients present in a typical Mediterranean diet have shown a positive impact on the biomarkers of inflammation, DNA damage, oxidative stress and genetic alterations, all factors that can influence $\mathrm{BC}$ outcomes. In addition, several studies have reported that a high adherence to MD is associated with a lower risk of the incidence of BC in post-menopausal women (41). A prospective cohort study conducted in the Mediterranean included 10,713 middle-aged, Spanish female university graduates and revealed an inverse association between the total polyphenol intake and the risk of BC for post-menopausal women (42).

The intake of polyphenols from food or as a food supplement for the prevention of BC is actually controversial, as only high concentrations are able to inhibit the proliferation of $\mathrm{ER} \alpha$ and $\mathrm{ER} \alpha^{+} \mathrm{BC}$ cells, while lower concentrations can even stimulate the growth of $E R \alpha^{+}$cells (43). One of the polyphenols studied for its inhibitory effects on BC is carnosol. An in vivo study demonstrated that this compound can reduce the proliferation of BC cell lines (MDA-MB-231) and can significantly inhibit invasion and metastasis both in vitro and in vivo; in particular, it has been shown that carnosol exerts its effect against BC through the downregulation of MMP-9 activity and expression, and by the inhibition of the STAT3 signaling pathway through the ROS-dependent proteasome degradation of the STAT3 protein (44).

The antioxidant properties of RES, a natural component of plants such as peanuts, cocoa, grapes, berries and red wine, are attributed to its polyphenolic stilbene structure. RES and its analogues have been classified as phytoestrogens able to bind estrogen receptor, and the results of investigations in ER-positive subtypes strongly suggest their use in hormone anticancer therapy. In fact, the majority of authors agree on the ability of RES to modulate $\operatorname{ER} \alpha$ and p53 expression in ER-positive BC: It inhibits the expression of major cell cycle-related genes, through the downregulation of ER $\alpha$ mRNA transcription. Recently, a membrane receptor site for RES on an integrin has been revealed in both ER-positive and ER-negative BC subtypes: Upon binding of RES with this receptor, the p53-dependent induction of apoptosis occurs (45). 
Therefore, RES and other stilbene derivatives would elicit significant cytotoxic and pro-apoptotic effects in ER-negative and 'triple-negative' breast cancer (TNBC) cells, lacking receptors for estrogens, progesterone and human epidermal growth factor. While the potential effects against BC of RES and other polyphenols such as curcumin (CUR), genistein, quercetin and silibinin have been widely investigated, interest towards the potential anticancer activity of luteolin is more recent. The mechanisms considered to be responsible for the cancer preventive activity of luteolin are reduced DNA alterations, antioxidant, anti-inflammatory and antiestrogenic action. Moreover, pro-apoptotic (e.g., by inhibiting PI3K/Akt and inducing FOXO3a activation) and chemosensitizing (mainly through JNK activation) effect may confer it a therapeutic potential. Luteolin inhibits BC cell survival, proliferation and migration and reduces angiogenesis, by modulating multiple signaling pathways and miRNAs (46).

EGCG and oleuropein (OLE) have been suggested as potential supplements against BC. Zan et al demonstrated that EGCG blocked cell cycle progression at the $\mathrm{G} 2 / \mathrm{M}$ phase in MCF-7. EGCG also induced apoptosis by inhibiting miR-25 expression and increasing PARP and pro-caspase expression (47). Oleuropein (OLE), a natural polyphenol, has also shown potential apoptotic and anti-invasive effects on MCF-7 cells. In fact, OLE reduced neoplastic cell invasiveness and viability and at the same time induced apoptosis in MCF-7 cancer cells (48); in particular, OLE acts on cancer control by epigenetic mechanism, such as the inhibition of histone deacetylase (HDAC) (49).

Finally, an in vitro study reported the anti-proliferative and cytotoxic effects of a polyphenol complex (catechin and lysine, 1:2) in BC cell lines. In particular, it was shown that this complex acts by interfering with glucose uptake and lactate production by tumor cells. It exerts selective anti-migratory (mediated by JAK2/STAT3 and Wnt pathway inhibition) and pro-apoptotic effects in breast, pancreatic and colorectal cancer cell lines (50). Nonetheless, epidemiological studies on flavonoids and BC have some limitations: Study design, a low sample size, variable doses of flavonoid intake and BC subtype are the most common (51).

\section{Lung cancer}

Lung cancer (LC) is the second most common type of cancer among both sexes, with a high mortality rate worldwide (52). Small cell (SCLC) and non-small cell LC (NSCLC) represent approximately 15 and $85 \%$ of all cases, respectively.

The consumption of fruit, vegetables and natural products is considered useful in the prevention and fight against LC. Some natural polyphenols have potential anticancer activities owing to their anti-proliferative, anti-migratory, anti-metastasis, anti-angiogenic and pro-apoptotic properties. A recent comprehensive review summarized preclinical studies investigating the molecular mechanisms of natural polyphenols or analogs with a potential role in LC. RES, CUR and EGCG emerged as the most studied compounds (53).

Lately, research has made immense progress in the comprehension of the mechanisms through which RES inhibits cell proliferation, induces apoptosis and cell cycle arrest, and suppresses invasion and metastasis. In particular,
RES has been shown to induce apoptosis through multiple signaling pathways, including the kinases, AKT, STAT3, PKC, p38, JNK, ERK, AMPK and PFK; various cyclins (A, D, E and $\mathrm{CDK}$ ) also act as cell cycle regulators. Multiple growth and transcription factors are also involved, as VEGF, FGF, TGF $\beta$, EGFR, AhR, Nrf2, NF- $\mathrm{B}$ and p53. The majority of these pathways have been identified to play a role in the pro-apoptotic effects of CUR and EGCG. Moreover, several studies have shown that modulation of miRNAs represent a key mechanism for the antitumor activity of RES in LC: $>70$ miRNAs related to apoptosis, cell cycle and differentiation have exhibited considerable changes in their expression levels in RES-treated A549 cells (53). CUR also has been shown to exert an anticancer effect in LC through epigenetic alterations and the regulation of miRNA expression (52).

Although the induction of apoptosis appears to be the main mechanism underlying the antitumor activities of polyphenols in LC, there is evidence of other mechanisms being involved in their inhibitory effects on lung tumor survival and progression. RES has been shown to lead to a significant reduction and imbalance in the pools of deoxyribonucleosides triphosphates (dNTP), which suppress subsequent DNA synthesis. Furthermore, the inhibition of DNA synthesis blocks the progression through the $\mathrm{S}$ phase in A549 cells, which can partially contribute to the cytotoxic effect of RES (54).

In vivo and in vitro studies have demonstrated that EGCG, a polyphenol present in green tea, inhibits the proliferation and migration and induces apoptosis in LC A549 and H1299 cells. These effects are partially achieved through inhibition of the NF- $\kappa \mathrm{B}$ signaling pathway. Furthermore, the concomitant administration of EGCG and BAY11-7082 has a synergistic effect and may serve as a new therapeutic strategy for LC. The anti-proliferative activity of EGCG seems to be attributable to suppressed phosphorylation of EGFR, ALK, ROS1, and in turn their downstream proteins, Akt and ERK; growth inhibition in xenograft tumors was associated with reduced HIF-1_ expression and tumor angiogenesis, suggesting that tumor response to EGCG is influenced by the tumor microenvironment $(53,55)$.

Dieckol is a polyphenolic substance extracted from brown algae. An in vitro study performed by Wang et al has shown that this substance has anticancer properties; in particular it acts by inhibiting the invasive and migratory properties of A549 cells and also by inducing apoptosis through inhibition of $\mathrm{PI} 3 \mathrm{~K} / \mathrm{AKT} / \mathrm{mTOR}$ signaling, activating the E-cadherin tumor suppressor protein. This suggests that dieckol may be a potent natural anticancer drug for the treatment of NSCLC (56). These data confirm the potential complementary role of RES, CUR and EGCG in cancer treatment to enhance the efficacy of existing therapies, reducing side-effects.

\section{Bladder cancer}

Polyphenols, including EGCG, CUR and RES, are known to exert an antioxidant effect; however, under certain conditions, they can be genotoxic for tumor cells. A study conducted by Almeida et al (57) evaluated the antitumor activity of RES and its possible mechanisms of action in bladder cancer cells with a different state of the TP53 gene. This gene responds to stress signals inducing cell cycle arrest, apoptosis, senescence 
and DNA repair; its mutations are the most common alterations in bladder cancer cells and are correlated with poor prognosis and recurrence. Its antitumor activity was evaluated in different bladder tumor cells (RT4, grade 1, TP53 wild type; 5637-grade 2; T24-grade 3, TP53 mutated). RES decreased cell proliferation and induced DNA damage in all neoplastic cell lines. However, TP53 wild-type cells were more resistant, while they were more prone to apoptosis, accompanied by AKT, mTOR, and SRC downregulation as well as modulation of the DNMT1 gene. Conversely, the prevalent action in TP53 mutated cells was cell cycle arrest at S phase with PLK1 downregulation, as well as modulation of the HOXB3/RASSF1A pathway and nuclear PCNA reduction in the highest-grade cells.

An in vitro study conducted on BFTC-905 cells, a human urinary bladder transitional cell carcinoma (TCC) cell, treated with EGCG, identified 108 differentially expressed genes and 22 candidate genes with potential miRNA interactions. These genes were mainly involved in the biogenesis of nicotinamide adenine dinucleotide (NAD), in the inflammatory response and in the oxidation-reduction metabolism (58). CUR is capable of suppressing the growth of a variety of cancer cells, including those of bladder cancer. CUR could be considered a promising candidate in bladder cancer therapy as it modulates various signaling pathways such as PI3K, Akt, mTOR and VEGF involved in the progression and malignancy of bladder cancer (59).

\section{Skin cancer}

Skin is considered the protective barrier of the organism, shielding it from harmful substances, mechanical damage, pathological invasion and radiation. Skin cancer (SC) is considered to be the most common type of cancer worldwide; this is the result of several mutations in cancer-related genes, including proto-oncogenes and tumor suppressors in skin cells, which cause an imbalance in cell homeostasis and excessive skin proliferation. The initiation of skin tumors can be attributed to various factors, although excessive exposure to UV radiation is considered the main risk factor for $\mathrm{SC}$. The main classification of SC is between melanoma and non-melanoma. Non-melanoma skin cancer (NMSC) in turn is distinguished into basal cell carcinoma (BCC) and squamous cell carcinoma (SCC) originated from epidermal keratinocytes. In a review aimed at elucidating the chemopreventive effects of polyphenols against SC metastasis, 34 in vivo mechanistic studies evaluating the efficacy of purified polyphenols were included; the authors of that study concluded that they exerted pro-apoptotic effects via the induction of ASK-1, Caspase-3, JNK-p38 and pRb; suppressed the cell cycle via the upregulation of $\mathrm{p} 21, \mathrm{Bcl} 2$, and $\mathrm{Bcl}-\mathrm{xL}$ and the downregulation of Bim, Bax, Puma and Noxa; reduced proliferation and angiogenesis through the upregulation of EGFR, MAPK, mTOR, PI3K/Akt, FAK/PTK2, JAK/STAT, VEGF and HIF-1 $\alpha$. The inhibition of cellular proliferation, invasion and metastasis would occur by modulating the expression of inflammatory and cytokine genes such as IL-6, IL-1, GM-CSF and TNF- $\alpha$, IL-2, GM-CSF and INF- $\gamma$ and IL-18 in melanoma models (60).

The treatment of melanoma remains a challenging issue, due to its aggressive metastatic ability and resistance to current therapeutic approaches. Though no clinical studies are yet available assessing the efficacy of the most active polyphenols in SC, epidemiological evidence indicates that the poorly soluble polyphenols, such as CUR, RES, EGCG, coumarin and quercetin, exert anticancer effects. In their review, Heenatigala Palliyage et al suggested that poorly soluble polyphenols, such as CUR, RES, quercetin, coumarin and EGCG may have significant benefits in the treatment of melanoma thanks to their antioxidant, anti-inflammatory, antiproliferative and chemoprotective properties. The well-known limitations to their use, i.e., low stability and poor bioavailability, may be overcome by using polyphenol-loaded nanotechnology-based delivery systems for topical and transdermal application, as polymeric nanoparticles, vesicles, lipid nanoparticles, nanofibers and nanohybrids (61).

Rodríguez-Luna et al (62) proposed the combination of the carotenoid fucoxanthin and the polyphenol rosmarinic acid (RA) as a natural promising tool in prevention of UVB-induced skin alterations as photo-aging, skin inflammation and its derivation to pre-cancerous lesions and skin carcinomas. RA is a phenolic ester traditionally isolated from some terrestrial plants as Rosmarinus officinalis L. or Melissa officinalis L. and also abundantly found in Zostera marina seagrass beds. RA has been widely studied due to its remarkable biological and pharmacological activities, including anti-microbial, antioxidant and anti-inflammatory properties. The authors of that study demonstrated that the combination of fucoxanthin and RA improved their antioxidant and anti-inflammatory profiles by reducing UVB-induced apoptosis and the consequent ROS production. This association also downregulated inflammasome components, such as NLRP3, ASC and caspase-1 and interleukin (IL)-1 production. Moreover, Nrf2 and HO-1 antioxidant genes expression increased in UVB-exposed pre-treated HaCaT cells.

The study conducted by Shin et al analyzed the effects of quercetin on skin aging. In particular, quercetin has been shown to suppress UV-induced matrix metalloproteinase-1 (MMP-1) and cyclooxygenase-2 (COX-2) expression by preventing UV-mediated collagen degradation in human skin tissues; inhibit the activator of protein-1 (AP-1) induced by $\mathrm{UV}$ rays and on $\mathrm{NF}-\kappa \mathrm{B}$; attenuate UV-ray phosphorylation of extracellular signal-regulated kinase (ERK), terminal kinases C-Jun N (JNK), protein kinase B (Akt) and signal transducer and activator of transcription 3 (STAT3); and to directly inhibit the kinase activity of the protein kinase $\mathrm{C} \delta(\mathrm{PKC} \delta)$ and JAK2 (63).

\section{Pancreatic cancer}

Pancreatic cancer is one of the most severe neoplasms due to a very poor prognosis, as in the majority of patients it is usually diagnosed at an advanced stage. Current chemotherapeutic agents administered to patients with metastasis pancreatic cancer are not able to significantly improve life expectancy. For this reason, novel therapeutic strategies are required to prevent or target metastatic disease aiming to improve the patient's outcome. Wei et al studied EGCG as a potentially safe and effective agent for use with gemcitabine in blocking the migration and invasion of pancreatic cancer, in part by inhibiting the Akt and EMT pathways. Since Akt signaling plays an important role in pancreatic cancer cell growth, 
Table II. Effects of polyphenols on cancer: Antitumor activity and signaling pathways.

\begin{tabular}{|c|c|c|c|}
\hline Cancer site & Polyphenols & Signaling pathways & Effects \\
\hline Prostate & $\begin{array}{l}\text { EGCG lycopene } \\
\text { resveratrol curcumin }\end{array}$ & $\begin{array}{l}\text { Matrix metalloproteinases } \\
\text { (MMP) And Their MMP } \\
\text { Tissue Inhibitor (TIMP) }\end{array}$ & $\begin{array}{l}\text { - Modulation of inflammatory pathways } \\
\text { - Induction of apoptosis and cycle arrest } \\
\text { - Influences epigenetic changes such as DNA } \\
\text { methylation and expression patterns of } \\
\text { microRNAs }\end{array}$ \\
\hline Breast & $\begin{array}{l}\text { EGCG lycopene } \\
\text { resveratrol curcumin } \\
\text { oleuropein }\end{array}$ & $\begin{array}{l}\mathrm{ER} \alpha \mathrm{ER} \alpha^{+} \\
\text {STAT3 } \\
\text { HER2 } \\
\text { MCF-7 }\end{array}$ & $\begin{array}{l}\text { - Inducing apoptosis and cycle arrest } \\
\text { - Modulation of inflammatory pathways } \\
\text { - Inhibition of STAT3 } \\
\text { - Inhibition of histone deacetylase }\end{array}$ \\
\hline Colon & $\begin{array}{l}\text { Resveratrol EGCG } \\
\text { ginkgetin }\end{array}$ & 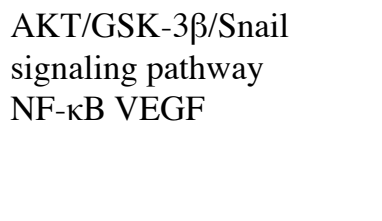 & $\begin{array}{l}\text { - AKT1 modulation epithelial mesenchymal } \\
\text { transition } \\
\text { - Inhibition of NF- } \mathrm{BB} \\
\text { - Reduction of genomic instability } \\
\text { - Inhibition of angiogenesis }\end{array}$ \\
\hline Lung & $\begin{array}{l}\text { RESVERATROL } \\
\text { CURCUMIN } \\
\text { ECGC }\end{array}$ & $\begin{array}{l}\text { NF- } \mathrm{B} \\
\mathrm{PI} 3 \mathrm{~K} / \mathrm{AKT} / \mathrm{mTOR}\end{array}$ & $\begin{array}{l}\text { - Modulation of inflammatory pathways } \\
\text { - Induction of apoptosis and cycle arrest } \\
\text { - Influences expression patterns of microRNAs } \\
\text { - Inhibition of the NF- } \mathrm{BB} \text { signaling pathway }\end{array}$ \\
\hline $\begin{array}{l}\text { Other types of cancer: } \\
\text { Bladder skin } \\
\text { pancreatic leukemia }\end{array}$ & $\begin{array}{l}\text { Resveratrol curcumin } \\
\text { ECGC quercetin }\end{array}$ & $\begin{array}{l}\text { COX-2 } \\
\text { MMP1 } \\
\text { DNTM1 gene } \\
\text { ERK } \\
\text { PFK } \\
\text { PI3K } \\
\text { AKT } \\
\text { mTOR } \\
\text { VEGF }\end{array}$ & $\begin{array}{l}\text { - Modulation of inflammatory pathways } \\
\text { - Induction of apoptosis and cycle arrest } \\
\text { - Influence expression patterns of microRNAs } \\
\text { - Inhibition of angiogenesis }\end{array}$ \\
\hline
\end{tabular}

downregulation of this kinase may partly explain the reduction in cell growth observed in EGCG-treated tumors. EGCG has effectively reduced pancreatic cancer cell growth in vitro and in vivo, otherwise there is little evidence of a beneficial effect of EGCG on cancer metastases (64).

\section{Leukemia}

Leukemia includes a group of hematological malignancies responsible for $8 \%$ of all cancers. the effectiveness of chemotherapy agents is low and the disease has an unfavorable prognosis with frequent recurrences and high mortality. Though the in vitro anti-leukemic effect of polyphenols has been studied for decades, there are only few in vivo studies.

RES has pleiotropic benefits due to its antioxidant and anti-inflammatory properties; recent findings suggest that it may have great potential in adjuvant therapy for leukemia. RES can act as an autophagy modulator and apoptosis inducer in MOLT-4 and HL-60 human leukemia cells (65). It has also been shown to reduce the therapeutic doses of drugs, such as barasertib and everolimus, minimizing their side-effects on both leukemic and normal lymphocytes (66).

RES has also been shown to be effective in reducing drug resistance: In HL-60/ADR cells, this occurs through the regulation of the PI3K/Akt/Nrf2 signaling pathway and MRP1 expression (67); in combination with prednisolone, in a dose-dependent manner, it reduces the expression of the MDR1 protein (68).

Recent findings suggest that CUR, in addition to its antioxidant and anti-inflammatory effects, may be a promising candidate for acute myeloid leukemia therapy (69). A recent study provided in vivo evidence confirming its effect on the apoptosis and invasion of human acute leukemia SHI-1 cells, through activation of JNK and p38 and inhibition of ERK and $\mathrm{NF}-\kappa \mathrm{B}$ signals. Furthermore, CUR may also downregulate the expression of MMP9 and MMP2 as well as vimentin, leading to suppression of the metastatic potency of SHI-1 (70).

EGCG exerts potent antitumor activity in hematological malignancies, including several types of leukemia. It induces apoptosis in chronic myeloid leukemia cells by regulating Bcr/Abl-mediated p38-MAPK/JNK and JAK2/STAT3/AKT signaling pathways (71).

\section{Conclusion}

There is a rich body of evidence suggesting that a diet rich in fruits and vegetables, mostly owing to the contribution of natural polyphenols, can reduce the incidence of specific 
cancers. Among the most extensively studied polyphenols are RES, EGCG and CUR. Many of the effects attributed to these compounds are linked to their antioxidant and anti-inflammatory properties; however, the multiple mechanisms involved include the modulation of molecular events and signaling pathways associated with cell survival, proliferation, differentiation, migration, angiogenesis, hormonal activities, detoxification enzymes and immune responses (Table II) (72-75).

Future research directions can potentially expand upon the use of dietary-based polyphenols as whole foods, whole-food extracts or purified compounds, especially in combinations, as a potent and effective method in cancer prevention and adjuvant therapy.

Notwithstanding their promising role in cancer prevention and treatment, polyphenols often have a poor bioavailability when administered as pure active principles, representing an important limit to their use. Possible interactions with other natural compounds present in the diet can also influence their efficacy. However, the bioavailability and thus efficacy of these compounds can be improved by the administration in combination with other phytochemicals, with anti-cancer drugs or in polyphenol-loaded nanotechnology-based delivery systems (50).

Particular attention should be paid to the safety of polyphenols. It should be noted that some isoflavones, such as genistein and daidzein, seem to have hormone-related adverse effects on cancer. Therefore, the use of these polyphenols in the treatment of cancer should be prudent.

Research interest on the effects of dietary polyphenols on the intestinal microbiota and the relative mechanisms of action has only recently spread. Dietary polyphenols seem to affect gut microbiota thanks to a bi-directional relation. On the one hand polyphenols can modulate the composition of microbiota; on the other hand, the microbiota is able to metabolize them into bioactive compounds. However, it seems clear that correct eating habits, typical of the Mediterranean diet, characterized by a high intake of polyphenols, play an important role in the maintenance of intestinal functions (76).

Recently, significant steps forward have been made in the understanding of the alterations driving to cancer development and progression at a cellular, molecular and genetic level. Relevant advances have been achieved also in the comprehension of the molecular mechanisms explaining the chemopreventive properties of specific polyphenols $(45,53)$.

Nonetheless, evidence from human studies is still inadequate and trials often resulted inconclusive or discordant. The main weaknesses of the studies conducted so far are the imprecise concentration of polyphenols in the tested foods or drinks, poor awareness of their kinetics and of the actual contribution of single compounds to their effect. Therefore, further clinical studies are warranted to support the use of polyphenols in the prevention and treatment of cancer.

\section{Acknowledgements}

Not applicable.

\section{Funding}

No funding was received.

\section{Availability of data and materials}

Not applicable.

\section{Authors' contributions}

GB, CC and CF substantially contributed to the conception and design of the study, revising it critically and providing final approval. MP, FG and SC were involved in the drafting, correction and revising the manuscript. All authors read and approved the final manuscript.

\section{Ethics approval and consent to participate}

Not applicable.

\section{Patient consent for publication}

Not applicable.

\section{Competing interests}

The authors declare that they have no competing interests.

\section{References}

1. Huang WY, Cai YZ and Zhang Y: Natural phenolic compounds from medicinal herbs and dietary plants: Potential use for cancer prevention. Nutr Cancer 62: 1-20, 2010.

2. Sharma A, Kaur M, Katnoria JK and Nagpal AK: Polyphenols in food: Cancer prevention and apoptosis induction. Curr Med Chem 25: 4740-4757, 2018.

3. Filetti V, Falzone L, Rapisarda V, Caltabiano R, Eleonora Graziano AC, Ledda C and Loreto C: Modulation of microRNA expression levels after naturally occurring asbestiform fibers exposure as a diagnostic biomarker of mesothelial neoplastic transformation. Ecotoxicol Environ Saf 198: 110640 , 2020.

4. Fenga C, Gangemi S, Di Salvatore V, Falzone L and Libra M: Immunological effects of occupational exposure to lead (Review). Mol Med Rep 15: 3355-3360, 2017.

5. Falzone L, Marconi A, Loreto C, Franco S, Spandidos DA and Libra M: Occupational exposure to carcinogens: Benzene, pesticides and fibers (Review). Mol Med Rep 14: 4467-4474, 2016.

6. Mazzocchi A, Leone L, Agostoni C and Pali-Schöll I: The secrets of the mediterranean diet. Does [only] olive oil matter? Nutrients 11: 2941, 2019.

7. Shaikh AA, Braakhuis AJ and Bishop KS: The mediterranean diet and breast cancer: A personalised approach. Healthcare (Basel) 7: 104, 2019.

8. Filippini T, Malavolti M, Borrelli F, Izzo AA, Fairweather-Tait SJ, Horneber M and Vinceti M: Green tea (Camellia sinensis) for the prevention of cancer. Cochrane Database Syst Rev 3: CD005004, 2020.

9. Blesso CN: Dietary anthocyanins and human health. Nutrients 11: 2107, 2019.

10. Nasir A, Bullo MMH, Ahmed Z, Imtiaz A, Yaqoob E, Jadoon M, Ahmed H, Afreen A and Yaqoob S: Nutrigenomics: Epigenetics and cancer prevention: A comprehensive review. Crit Rev Food Sci Nutr 60: 1375-1387, 2020.

11. Zhou Y, Zheng J, Li Y, Xu DP, Li S, Chen YM and Li HB: Natural polyphenols for prevention and treatment of cancer. Nutrients 8 : $515,2016$.

12. Khan $\mathrm{N}$ and Mukhtar H: Tea polyphenols in promotion of human health. Nutrients 11: 39, 2018.

13. Giordano A and Tommonaro G: Curcumin and cancer. Nutrients 11: 2376, 2019.

14. Han Y, Huang M, Li L, Cai X, Gao Z, Li F, Rakariyatham K, Song M, Fernández Tomé S and Xiao H: Non-extractable Polyphenols from cranberries: Potential anti-inflammation and anti-colon-cancer agents. Food Funct 10: 7714-7723, 2019. 
15. Yao Y, Wang $\mathrm{H}, \mathrm{Xu} \mathrm{F}$, Zhang $\mathrm{Y}$, Li Z, Ju X and Wang L: Insoluble-bound polyphenols of adlay seed ameliorate $\mathrm{H}(2)$ $\mathrm{O}(2)$-induced oxidative stress in $\mathrm{HepG} 2$ cells via $\mathrm{Nrf} 2$ signalling. Food Chem 325: 126865, 2020.

16. Yahfoufi N, Alsadi N, Jambi M and Matar C: The immunomodulatory and anti-inflammatory role of polyphenols. Nutrients 10 : 1618,2018

17. Singh SK, Banerjee S, Acosta EP, Lillard JW and Singh R: Resveratrol induces cell cycle arrest and apoptosis with docetaxel in prostate cancer cells via a p53/ p21WAF1/CIP1 and p27KIP1 pathway. Oncotarget 8: 17216-17228, 2017.

18. Tsoupras AB, Iatrou C, Frangia $\mathrm{C}$ and Demopoulos $\mathrm{CA}$ : The implication of platelet activating factor in cancer growth and metastasis: Potent beneficial role of PAF-inhibitors and antioxidants. Infect Disord Drug Targets 9: 390-399, 2009.

19. Hubbard GP, Wolffram S, Lovegrove JA and Gibbins JM: Ingestion of quercetin inhibits platelet aggregation and essential components of the collagen-stimulated platelet activation pathway in humans. J Thromb Haemost 2: 2138-2145, 2004.

20. Tzeng SH, Ko WC, Ko FN and Teng CM: Inhibition of platelet aggregation by some flavonoids. Thromb Res 64: 91-100, 1991.

21. Fragopoulou E, Nomikos T, Karantonis HC, Apostolakis C, Pliakis E, Samiotaki M, Panayotou G and Antonopoulou S: Biological activity of acetylated phenolic compounds. J Agric Food Chem 55: 80-89, 2007.

22. Mouria M, Gukovskaya AS, Jung Y, Buechler P, Hines OJ, Reber HA and Pandol SJ: Food-derived polyphenols inhibit pancreatic cancer growth through mitochondrial cytochrome $\mathrm{C}$ release and apoptosis. Int J Cancer 98: 761-769, 2002.

23. Padmanabhan S, Waly MI, Taranikanti V, Guizani N, Ali A, Rahman MS, Al-Attabi Z, Al-Malky RN, Al-Maskari SNM, Al-Ruqaishi BRS, et al: Folate/vitamin B12 supplementation combats oxidative stress-associated carcinogenesis in a rat model of colon cancer. Nutr Cancer 71: 100-110, 2019.

24. Sánchez-Tapia M, Tovar AR and Torres N: Diet as regulator of gut microbiota and its role in health and disease. Arch Med Res 50: 259-268, 2019.

25. Concettina F, Chiara C, Emanuela C, Luigi R, Carmela A, Silvia G, Anca DO and Michael TA: Current evidence on the protective effect of dietary polyphenols on breast cancer. Farmacia 64: 1, 2016.

26. Costa C, Tsatsakis A, Mamoulakis C, Teodoro M, Briguglio G, Caruso E, Tsoukalas D, Margina D, Dardiotis E, Kouretas D and Fenga C: Current evidence on the effect of dietary polyphenols intake on chronic diseases. Food Chem Toxicol 110: 286-299, 2017.

27. Russo GI, Solinas T, Urzì D, Privitera S, Campisi D, Cocci A, Carini M, Madonia M, Cimino S and Morgia G: Adherence to Mediterranean diet and prostate cancer risk in Sicily: Population-based case-control study. Int J Impot Res 31: 269-275, 2019.

28. Livingstone TL, Beasy G, Mills RD, Plumb J, Needs PW, Mithen $\mathrm{R}$ and Traka MH: Plant bioactives and the prevention of prostate cancer: Evidence from human studies. Nutrients 11: 2245, 2019.

29. Pejčić T, Tosti T, Džamić Z, Gašić U, Vuksanović A, Dolićanin Z and Tešić Ž: The polyphenols as potential agents in prevention and therapy of prostate diseases. Molecules 24: 3982, 2019.

30. Jang YG, Go RE, Hwang KA and Choi KC: Resveratrol inhibits DHT-induced progression of prostate cancer cell line through interfering with the AR and CXCR4 pathway. Steroid Biochem Mol Biol 192: 105406, 2019.

31. Deb G, Shankar E, Thakur VS, Ponsky LE, Bodner DR, Fu P and Gupta S: Green tea-induced epigenetic reactivation of tissue inhibitor of matrix metalloproteinase-3 suppresses prostate cancer progression through histone-codifying enzymes. Mol Carcinog 58: 1194-1207, 2019.

32. Lee CW, Chen HJ, Xie GR and Shih CK: Djulis (Chenopodium Formosanum) prevents colon carcinogenesis via regulating antioxidative and apoptotic pathways in rats. Nutrients 11: 2168, 2019.

33. Vivarelli S, Salemi R, Candido S, Falzone L, Santagati M, Stefani S, Torino F, Banna GL, Tonini G and Libra M: Gut microbiota and cancer: From pathogenesis to therapy. Review Cancers (Basel) 11: 38, 2019

34. Cueva C, Silva M, Pinillos I, Bartolomé B and Moreno-Arribas MV: Interplay between dietary polyphenols and oral and gut microbiota in the development of colorectal cancer. Nutrients 12: 625, 2020
35. Zhao Y and Zhang X: Interactions of tea polyphenols with intestinal microbiota and their implication for anti-obesity. J Sci Food Agric 100: 897-903, 2020.

36. $\mathrm{Li}$ Y, Gao X and Lou Y: Interactions of tea polyphenols with intestinal microbiota and their implication for cellular signal conditioning mechanism. J Food Biochem 43: e12953, 2019.

37. Yuan L, Zhou M, Huang D, Wasan HS, Zhang K, Sun L, Huang H, Ma S, Shen M and Ruan S: Resveratrol inhibits the invasion and metastasis of colon cancer through reversal of epithelial-mesenchymal transition via the AKT/GSK-3 $\beta /$ Snail signaling pathway. Mol Med Rep 20: 2783-2795, 2019.

38. Chen T, Shi N and Afzali A: Chemopreventive effects of strawberry and black raspberry on colorectal cancer in inflammatory bowel disease. Nutrients 11: 1261, 2019.

39. Hu WH, Chan GK, Duan R, Wang HY, Kong XP, Dong TT and Tsim KW: Synergy of Ginkgetin and resveratrol in suppressing VEGF-induced angiogenesis: A therapy in treating colorectal cancer. Cancers (Basel) 11: 1828, 2019.

40. Concettina F: Occupational exposure and risk of breast cancer. Biomed Rep 4: 282-292, 2016.

41. Kwon YJ, Cho YE, Cho AR, Choi WJ, Yun S, Park H, Kim HS, Cashion AK, Gill J, Lee H and Lee JW: The possible influence of mediterranean diet on extracellular vesicle miRNA expression in breast cancer survivors. Cancers (Basel) 12: 1355, 2020.

42. Gardeazabal I, Romanos-Nanclares A, Martínez-González MÁ, Sánchez-Bayona R, Vitelli-Storelli F, Gaforio JJ, Aramendía-Beitia JM and Toledo E: Total polyphenol intake and breast cancer risk in the Seguimiento Universidad De Navarra (SUN) cohort. Br J Nutr 122: 542-551, 2019.

43. Poschner S, Maier-Salamon A, Thalhammer T and Jäger W: Resveratrol and other dietary polyphenols are inhibitors of estrogen metabolism in human breast cancer cells. J Steroid Biochem Mol Biol 190: 11-18, 2019.

44. Alsamri H, El Hasasna H, Al Dhaheri Y, Eid AH, Attoub S and Iratni R: Carnosol, a natural polyphenol, inhibits migration, metastasis, and tumor growth of breast cancer via a ROS-dependent proteasome degradation of STAT3. Front Oncol 9: 743, 2019.

45. Horgan XJ, Tatum H, Brannan E, Paull DH and Rhodes LV: Resveratrol analogues surprisingly: Effective against triple-negative breast cancer, independent of ER $\alpha$. Oncol Rep 41: 3517-3526, 2019.

46. Ahmed S, Khan H, Fratantonio D, Hasan MM, Sharifi S, Fathi N, Ullah H and Rastrelli L: apoptosis induced by luteolin in breast cancer: Mechanistic and therapeutic perspectives. Phytomedicine 59: 152883, 2019.

47. Zan L, Chen Q, Zhang L and Li X: Epigallocatechin gallate (EGCG) suppresses growth and tumorigenicity in breast cancer cells by downregulation of miR-25. Bioengineered 10: 374-382, 2019.

48. Mansouri N, Alivand MR, Bayat S, Khaniani MS and Derakhshan SM: The hopeful anticancer role of oleuropein in breast cancer through histone deacetylase modulation. J Cell Biochem 120: 17042-17049, 2019.

49. Bayat S, Mansoori Derakhshan S, Mansoori Derakhshan N, Shekari Khaniani M and Alivand MR: Downregulation of HDAC2 and HDAC3 via oleuropein as a potent prevention and therapeutic agent in MCF-7 breast cancer cells. J Cell Biochem 120: 9172-9180, 2019.

50. Silva C, Correia-Branco A, Andrade N, Ferreira AC, Soares ML, Sonveaux P, Stephenne J and Martel F: Selective pro-apoptotic and antimigratory effects of polyphenol complex catechin:lysine 1:2 in breast, pancreatic and colorectal cancer cell lines. Eur J Pharmacol 859: 172533, 2019.

51. Selvakumar P, Badgeley A, Murphy P, Anwar H, Sharma U, Lawrence K and Lakshmikuttyamma A: Flavonoids and Other Polyphenols Act as Epigenetic Modifiers in Breast Cancer Nutrients 12: 761, 2020.

52. Wan Mohd Tajuddin WNB, Lajis NH, Abas F, Othman I and Naidu R: Mechanistic understanding of curcumin's therapeutic effects in lung cancer Nutrients 11: 2989, 2019.

53. Zhou Q, Pan H and Li J: Molecular insights into potential contributions of natural polyphenols to lung cancer treatment. Cancers (Basel) 11: 1565, 2019

54. Amararathna M, Hoskin DW and Rupasinghe HPV: Anthocyanin-rich haskap (Lonicera Caerulea L.) berry extracts reduce nitrosamine-induced DNA damage in human normal lung epithelial cells in vitro. Food Chem Toxicol 141: $111404,2020$. 
55. Zhang L, Xie J, Gan R, Wu Z, Luo H, Chen X, Lu Y, Wu L and Zheng D: Synergistic Inhibition of Lung Cancer Cells by EGCG and NF- $\kappa$ B Inhibitor BAY11-7082. J Cancer 10: 6543-6556, 2019.

56. Wang CH, Li XF, Jin LF, Zhao Y, Zhu GJ and Shen WZ: Dieckol inhibits non-small-cell lung cancer cell proliferation and migration by regulating the PI3K/AKT signaling pathway. J Biochem Mol Toxicol 33: e22346, 2019.

57. Almeida TC, Guerra CCC, De Assis BLG, de Oliveira Aguiar Soares RD, Garcia CCM, Lima AA and da Silva GN Antiproliferative and toxicogenomic effects of resveratrol in bladder cancer cells with different TP53 status. Environ Mol Mutagen 60: 740-751, 2019.

58. Lee HY, Chen YJ, Chang WA, Li WM, Ke HL, Wu WJ and Kuo PL: Effects of epigallocatechin gallate (EGCG) on urinary bladder urothelial carcinoma-next-generation sequencing and bioinformatics approaches. Medicina (Kaunas) 55: 768, 2019.

59. Ashrafizadeh M, Yaribeygi $\mathrm{H}$ and Sahebkar A: Therapeutic effects of curcumin against bladder cancer: A review of possible molecular pathways. Anticancer Agents Med Chem 20: 667-677, 2020.

60. Sajadimajd S, Bahramsoltani R, Iranpanah A, Kumar Patra J,Das G Gouda S, Rahimi R, Rezaeiamiri E, Cao H, Giampieri F, et al: Advances on natural polyphenols as anticancer agents for skin cancer. Pharmacol Res 151: 104584, 2020.

61. Heenatigala Palliyage G, Singh S, Ashby CR Jr, Tiwari AK and Chauhan H: Pharmaceutical topical delivery of poorly soluble polyphenols: Potential role in prevention and treatment of melanoma. AAPS PharmSciTech 20: 250, 2019.

62. Rodríguez-Luna A, Ávila-Román J, Oliveira H, Motilva V and Talero E: Fucoxanthin and rosmarinic acid combination has anti-inflammatory effects through regulation of NLRP3 inflammasome in UVB-exposed HaCaT keratinocytes. Mar Drugs 17: 451, 2019.

63. Shin EJ, Lee JS, Hong S, Lim TG and Byun S: Quercetin directly targets JAK2 and PKC $\delta$ and prevents UV-induced photoaging in human skin. Int J Mol Sci 20: 5262, 2019.

64. Wei R, Penso NEC, Hackman RM, Wang Y and Mackenzie GG: Epigallocatechin-3-gallate (EGCG) suppresses pancreatic cancer cell growth, invasion, and migration partly through the inhibition of Akt pathway and epithelial-mesenchymal transition: Enhanced efficacy when combined with gemcitabine. Nutrients 11: 1856, 2019.

65. Siedlecka-Kroplewska K, Wozniak M and Kmiec Z: The wine polyphenol resveratrol modulates autophagy and induces apoptosis in MOLT-4 and HL-60 human leukemia cells. J Physiol Pharmacol 70: 825-838, 2019.

66. Ivanova D, Zhelev Z, Semkova S, Aoki I and Bakalova R Resveratrol modulates the redox-status and cytotoxicity of anticancer drugs by sensitizing leukemic lymphocytes and protecting normal lymphocytes. Anticancer Res 39: 3745-3755, 2019.

67. Li Y, Guo Y, Feng Z, Bergan R, Li B, Qin Y, Zhao L, Zhang Z and Shi M: Involvement of the PI3K/Akt/Nrf2 signaling pathway in resveratrol-mediated reversal of drug resistance in HL-60/ADR cells. Nutr Cancer 71: 1007-1018, 2019.
68. Talebi M, Bahar Aghdam S, Azimi A, Mohammadi H, Karimi Yonjali S, Asariha M and Zadi Heydarabad M: Regulatory effect of resveratrol and prednisolone on MDR1 protein expression in acute lymphoblastic leukemia cell line (CCRF-CEM). Asian Pac J Cancer Prev 20: 1171-1176, 2019.

69. Kouhpeikar H, Butler AE, Bamian F, Barreto GE, Majeed M and Sahebkar A: Curcumin as a therapeutic agent in leukemia. J Cell Physiol 234: 12404-12414, 2019.

70. Zhu G, Shen Q, Jiang H, Ji O, Zhu L and Zhang L: Curcumin inhibited the growth and invasion of human monocytic leukaemia SHI-1 cells in vivo by altering MAPK and MMP signalling. Pharm Biol 58: 25-34, 2020.

71. Xiao X, Jiang K, Xu Y, Peng H, Wang Z, Liu S and Zhang G: (-)-Epigallocatechin-3-gallate induces cell apoptosis in chronic myeloid leukaemia by regulating Bcr/Abl-mediated p38-MAPK/JNK and JAK2/STAT3/AKT signalling pathways. Clin Exp Pharmacol Physiol 46: 126-136, 2019.

72. Neagu M, Constantin C, Popescu ID, Zipeto D, Tzanakakis G, Nikitovic D, Fenga C, Stratakis CA, Spandidos DA and Tsatsakis AM: Inflammation and metabolism in cancer cell-mitochondria key player. Front Oncol 9: 348, 2019.

73. McCubrey JA, Rakus D, Gizak A, Steelman LS, Abrams SL, Lertpiriyapong K, Fitzgerald TL, Yang LV, Montalto G, Cervello M, et al: Effects of mutations in Wnt $/ \beta$-catenin, hedgehog, Notch and PI3K pathways on GSK-3 activity-Diverse effects on cell growth, metabolism and cancer. Biochim Biophys Acta 1863: 2942-2976, 2016.

74. Negrei C, Hudita A, Ginghina O, Galateanu B, Voicu SN, Stan M, Costache M, Fenga C, Drakoulis N and Tsatsakis AM: Colon cancer cells gene expression signature as response to 5-fluorouracil, oxaliplatin, and folinic acid treatment. Front Pharmacol 7: $172,2016$.

75. Steelman LS, Fitzgerald T, Lertpiriyapong K, Cocco L, Follo MY, Martelli AM, Neri LM, Marmiroli S, Libra M, Candido S, et al: Critical roles of EGFR family members in breast cancer and breast cancer stem cells: Targets for therapy. Curr Pharm Des 22: 2358-2388, 2016.

76. Peron G, Hidalgo-Liberona N, González-Domínguez R, Garcia-Aloy M, Guglielmetti S, Bernardi S, Kirkup B, Kroon PA, Cherubini A, Riso P and Andrés-Lacueva C: Exploring the molecular pathways behind the effects of nutrients and dietary polyphenols on gut microbiota and intestinal permeability: A perspective on the potential of metabolomics and future clinical applications. J Agric Food Chem 68: $1780-1789,2020$

This work is licensed under a Creative Commons Attribution-NonCommercial-NoDerivatives 4.0 International (CC BY-NC-ND 4.0) License. 